Supporting Information

\title{
Stereoselective Synthesis of Pyrrolidine Derivatives via Reduction of Substituted Pyrroles
}

\author{
Chao Jiang, Alison J. Frontier* \\ Department of Chemistry, University of Rochester, Rochester, New York, 14627 \\ frontier@chem.rochester.edu
}

\section{Table of Contents}

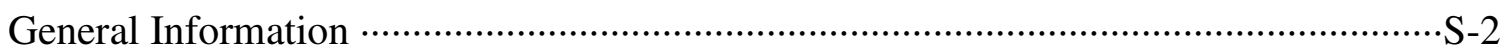

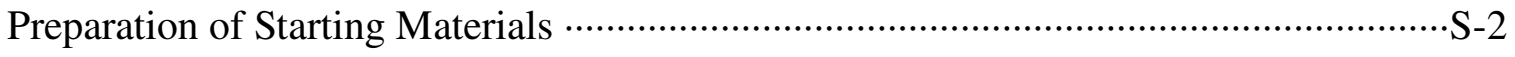

General Procedure for the Heterogeneous Hydrogenation ……..............................S-10

Tabulated Spectral Data for Hydrogenation Products ……...................................S-11

Intermediates Isolated During Mechanistic Studies …….......................................... 15

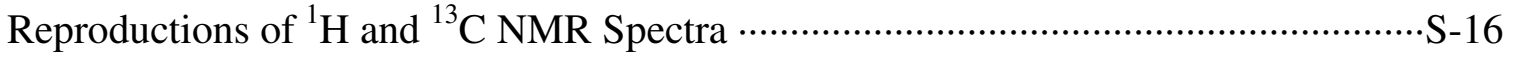




\section{General Information}

Reagents were used as obtained from commercial supplier without further purification. Reaction solvents were purchased from Fisher and dispensed using the Glass Contour solvent purification system. ACS grade hexanes and ethyl acetate (EtOAc) are used for column chromatography.

Thin layer chromatography (TLC) analysis was determined using precoated silica gel $60 \mathrm{~F}_{254}$ glass-supported plate (EMD product). Spots were visualized by UV light $(254 \mathrm{~nm})$, or by staining with potassium permanganate, $p$-anisaldehyde, ninhydrin or ceric ammonium molybdate solution followed by heating. Column chromatography separations were carried out on silica gel 60 (230-400 mesh) (EMD product). Fisherbrand PVDF Syringe filters $(0.22 \mu \mathrm{m})$ were used for microscale filtration.

${ }^{1} \mathrm{H}$ NMR spectra were recorded on either a $400 \mathrm{MHz}$ or a $500 \mathrm{MHz}$ Avance spectrometer with $\mathrm{CDCl}_{3}$ as the solvent and internal reference $(7.24 \mathrm{ppm}) .{ }^{13} \mathrm{C} \mathrm{NMR}$ spectra were recorded on Avance 75 or $125 \mathrm{MHz}$ with the central peak of $\mathrm{CDCl}_{3}(77.0 \mathrm{ppm})$ as the internal reference. Infrared spectra were recorded on a $8400 \mathrm{~S}$ Shimadzu FTIR spectometer using $\mathrm{NaCl}$ plates. High resolution mass spectra was done on a ThermoFinnigan MAT 95XL at the Chemistry Instrumentation Center of the University of Buffalo. X-ray analysis was performed at Department of Chemistry, University of Rochester X-ray facility.

\section{Preparation of Starting Materials}

General Three-Step Procedure for the Synthesis of Compounds 1a, 1b, 1g, 1h, 1j<smiles>[R7]C(N[B])C(=O)OCc1ccccc1</smiles>

S-1<smiles>[R]c1c(OC)ccn1[R7]</smiles>

S-3<smiles>[R2]C1C(OC)=CC(=O)N1[R1]</smiles>

S-2

Step 1. Addition/wittig olefination of $\alpha$-amino carboxylic acid methyl ester. ${ }^{1}$ A solution of

${ }^{1}$ Löffler, J.; Schobert, R. J. Chem. Soc., Perkin Trans. 1 1996, 2799-2802. 
methyl(triphenylphosphoranylidene)acetate $(10 \mathrm{mmol}, 1.25$ equiv. $)$ and $\alpha$-amino carboxylic acid methyl ester hydrochloride $\mathbf{S - 1}$ ( $8 \mathrm{mmol}, 1$ equiv.) in toluene (70 mL) was heated to reflux with exclusion of air and moisture for $12 \mathrm{~h}$. After cooling, the reaction mixture was evaporated on a rotary evaporator and the resulting residue was purified by column chromatography to give alkoxypyrrolinones $\mathbf{S - 2}$.

Step 2. DIBAL reduction of alkoxypyrrolinone. ${ }^{2}$ To a stirred solution of alkoxypyrrolinone S-2 (4.0 mmol) in $90 \mathrm{~mL}$ of THF at $0{ }^{\circ} \mathrm{C}$ was added $8.0 \mathrm{ml}(12 \mathrm{mmol}, 3$ equiv.) of $1.5 \mathrm{M}$ DIBAL in hexane dropwise over $2 \mathrm{~min}$. The mixture was allowed to warm slowly to room temperature and was stirred for $12 \mathrm{~h}$. It was then poured into $90 \mathrm{~mL}$ of cold $1 \mathrm{~N}$ aqueous $\mathrm{NaOH}$ and was extracted with three $90 \mathrm{~mL}$ portions of ether. The combined extract was dried over $\mathrm{MgSO}_{4}$, and concentrated. The residue was used in the next step without further purification because of the instability of the 3-alkoxypyrrole $\mathbf{S - 3}$.

Step 3. Acylation of 3-alkoxypyrrole. ${ }^{3}$ A solution of pyridine (3.29 mmol, 1.2 equiv.) in dry dichloromethane $(6 \mathrm{~mL})$ was added dropwise to a stirred solution of alkyl chlorooxoacetate (3.02 mmol, 1.1 equiv.) in dry dichloromethane $(6 \mathrm{~mL})$ at $-78^{\circ} \mathrm{C}$ under nitrogen. A solution of the 3-alkoxypyrrole $\mathbf{S - 3}$ (2.74 mmol, 1.0 equiv.) in dry dichloromethane (6 $\mathrm{mL})$ was added dropwise to it and the solution allowed to warm slowly to room temperature. After being stirred for $48 \mathrm{~h}$, the solution was washed with dilute hydrochloric acid, water and brine, dried $\left(\mathrm{MgSO}_{4}\right)$ and evaporated. The residue was purified by column chromatography to give the hydrogenation substrate $\mathbf{1}$.

\section{Preparation of compound 1a}<smiles>CCOC(=O)c1cc(OC)c2n1CCCC2</smiles>

1a was prepared by the general procedure from methyl pipecolinate hydrochloride $(3.6 \mathrm{~g}$, $20 \mathrm{mmol})$. Purification by column chromatography on silica gel, diethyl ether/hexane 1:1, gave $0.78 \mathrm{~g}$ of $\mathbf{1 a}\left(54 \%\right.$ yield $\left.^{4}\right) .{ }^{1} \mathrm{H}$ NMR $\left(400 \mathrm{MHz} \mathrm{CDCl}_{3}\right) \delta 6.83(\mathrm{~s}, 1 \mathrm{H}), 4.40-4.34(\mathrm{~m}$, 4H), $3.78(\mathrm{~s}, 3 \mathrm{H}), 2.74(\mathrm{t}, 2 \mathrm{H}, \mathrm{J}=6.4 \mathrm{~Hz}), 1.96(\mathrm{~m}, 2 \mathrm{H}), 1.84(\mathrm{~m}, 2 \mathrm{H}), 1.41(\mathrm{t}, 3 \mathrm{H}, \mathrm{J}=7.2$ $\mathrm{Hz}) ;{ }^{13} \mathrm{C}$ NMR $\left(500 \mathrm{MHz}, \mathrm{CDCl}_{3}\right) \delta 171.2,164.1,145.2,130.6,122.3,106.3,61.7,57.7$, 46.5, 23.1, 21.1, 18.8, 14.1; IR (neat) 2943, 1728, 1615, 1416, 1317, 1276, 1258, 1179, 1148, 1082, $747 \mathrm{~cm}^{-1}$; HRMS m/z calc. for $\mathrm{C}_{13} \mathrm{H}_{17} \mathrm{O}_{4} \mathrm{~N}\left(\mathrm{M}+\mathrm{H}^{+}\right)$252.1230, found 252.12299 .

\footnotetext{
${ }^{2}$ Kochhar, K. S.; Pinnick, H. W. J. Org. Chem. 1984, 49, 3222-3224.

${ }^{3}$ Harrison, C.-A.; Jackson, P. M.; Moody, C. J.; Williams, J. M. J. J. Chem. Soc., Perkin Trans. 1 1995, 1131-1136.

${ }^{4}$ Yield of the last two steps in the general procedures (DIBAL reduction of alkoxypyrrolinone and acylation of 3-alkoxypyrrole).
} 


\section{Preparation of compound $1 \mathrm{~b}$}<smiles>COC(=O)c1cc(OC)c2n1CCC2</smiles>

1b was prepared by the general procedure from proline methyl ester hydrochloride $(2.8 \mathrm{~g}$, $17 \mathrm{mmol}$ ). Purification by column chromatography on silica gel, diethyl ether/hexane $1: 1$, gave $0.35 \mathrm{~g}$ of $\mathbf{1 b}\left(32 \%\right.$ yield). ${ }^{1} \mathrm{H}$ NMR $\left(400 \mathrm{MHz}, \mathrm{CDCl}_{3}\right) \delta 6.95(\mathrm{~s}, 1 \mathrm{H}), 4.36(\mathrm{~m}, 2 \mathrm{H}, \mathrm{J}$ $=7.2 \mathrm{~Hz}), 3.91(\mathrm{~s}, 3 \mathrm{H}), 3.80(\mathrm{~s}, 3 \mathrm{H}), 2.89(\mathrm{t}, 2 \mathrm{H}, \mathrm{J}=7.2 \mathrm{~Hz}), 2.54(\mathrm{~m}, 2 \mathrm{H}) ;{ }^{13} \mathrm{C} \mathrm{NMR}$ $\left(500 \mathrm{MHz}, \mathrm{CDCl}_{3}\right) \delta 169.8,163.5,142.5,136.6,120.7,109.6,58.0,52.5,48.8,26.7,23.3$; IR (neat) 2953, 1729, 1616, 1557, 1456, 1428, 1410, 1265, 1152, 1053, 1009, 929, 814, 786, 769, $625 \mathrm{~cm}^{-1}$; HRMS $\mathrm{m} / z$ calc. for $\mathrm{C}_{11} \mathrm{H}_{13} \mathrm{O}_{4} \mathrm{~N}\left(\mathrm{M}+\mathrm{H}^{+}\right)$224.0917, found 224.09151 .

\section{Preparation of compound $1 c^{3}$}

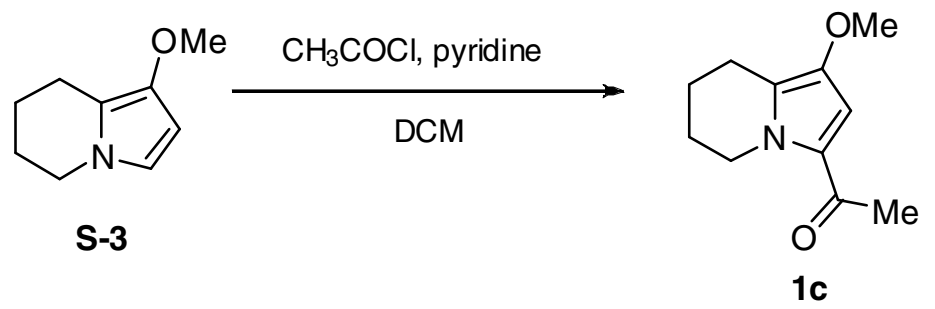

A solution of pyridine $(0.26 \mathrm{~g}, 3.29 \mathrm{mmol})$ in dry dichloromethane $(6 \mathrm{~mL})$ was added dropwise to a stirred solution of acetyl chloride $(0.24 \mathrm{~g}, 3.02 \mathrm{mmol})$ in dry dichloromethane $(6 \mathrm{~mL})$ at $-78^{\circ} \mathrm{C}$ under nitrogen. A solution of the pyrrole $\mathbf{S}-3^{5}(0.41 \mathrm{~g}$, $2.74 \mathrm{mmol})$ in dry dichloromethane $(6 \mathrm{~mL})$ was added dropwise to it and the solution allowed to warm slowly to room temperature. After being stirred for $48 \mathrm{~h}$, the solution was washed with dilute hydrochloric acid, water and brine, dried $\left(\mathrm{MgSO}_{4}\right)$ and evaporated. The residue was purified by column chromatography (silica gel, ethyl acetate/hexane 1:1) to give a $0.30 \mathrm{~g}\left(56 \%\right.$ yield) of the title compound 1c. ${ }^{1} \mathrm{H} \mathrm{NMR}\left(400 \mathrm{MHz}, \mathrm{CDCl}_{3}\right): 6.50$ (s, $1 \mathrm{H}), 4.32(\mathrm{t}, 2 \mathrm{H}, \mathrm{J}=6 \mathrm{~Hz}), 3.73(\mathrm{~s}, 3 \mathrm{H}), 2.68(\mathrm{t}, 2 \mathrm{H}, \mathrm{J}=6.4 \mathrm{~Hz}), 2.32(\mathrm{~s}, 3 \mathrm{H}), 1.88(\mathrm{~m}, 2 \mathrm{H})$, $1.77(\mathrm{~m}, 2 \mathrm{H}) ;{ }^{13} \mathrm{C} \mathrm{NMR}\left(500 \mathrm{MHz}, \mathrm{CDCl}_{3}\right) \delta 186.3,143.5,125.0,124.9,103.5,58.0,46.2$, 26.8, 23.4, 20.8, 19.2; IR (neat) $\mathrm{cm}^{-1}$ 2942, 1634, 1557, 1456, 1422, 1403, 1353, 1316, $1229,1187,1124,1106,993,933,780,604 ;$ HRMS $m / z$ calc. for $\mathrm{C}_{11} \mathrm{H}_{15} \mathrm{O}_{2} \mathrm{~N}\left(\mathrm{M}+\mathrm{H}^{+}\right)$ 194.1176, found 194.11794.

\section{Preparation of compound $1 d^{6}$}

\footnotetext{
${ }^{5}$ Pyrrole was prepared by the general procedure from methyl pipecolinate hydrochloride.

${ }^{6}$ Verniest, G.; Van Hende, E.; Surmont, R.; De Kimpe, N. Org. Lett. 2006, 8, 4767-4770.
} 


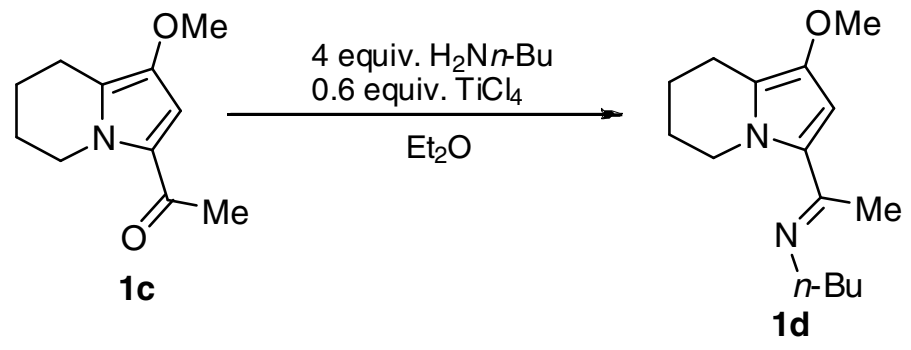

To a cooled $\left(0^{\circ} \mathrm{C}\right)$ solution of pyrrole $1 \mathrm{c}(65 \mathrm{mg}, 0.34 \mathrm{mmol})$ and butylamine $(98 \mathrm{mg}, 1.35$ mmol, 4 equiv.) in $2 \mathrm{ml}$ of dry diethyl ether is added titanium(IV) chloride (39mg, 0.20 mmol, 0.6 equiv.). The mixture is stirred for $15 \mathrm{~h}$ allowing the temperature to rise to room temperature. After completion of the reaction, the mixture is poured in a biphasic mixture of $20 \mathrm{ml}$ of aqueous $0.5 \mathrm{M} \mathrm{NaOH}$ solution and $10 \mathrm{ml}$ of diethyl ether. After separation of the organic phase, the aqueous phase is again extracted with $20 \mathrm{ml}$ diethyl ether (2 times). The combined organic phases are dried over a mixture of $\mathrm{MgSO}_{4}$ and $\mathrm{K}_{2} \mathrm{CO}_{3}$ (10:1). Evaporation of the solvent, the crude imine was purified by column chromatography (silica gel, ethyl acetate/hexane 1:1) to give a $74 \mathrm{mg}$ ( $88 \%$ yield) of the title compound $\mathbf{1 d} .{ }^{1} \mathrm{H}$ NMR (400 MHz, $\left.\mathrm{CDCl}_{3}\right): 6.21(\mathrm{~s}, 1 \mathrm{H}), 4.32(\mathrm{t}, 2 \mathrm{H}, \mathrm{J}=6 \mathrm{~Hz}), 3.75(\mathrm{~s}, 3 \mathrm{H}), 3.38(\mathrm{t}, 2 \mathrm{H}, \mathrm{J}=$ $6.8 \mathrm{~Hz}), 2.72(\mathrm{t}, 2 \mathrm{H}, \mathrm{J}=6.4 \mathrm{~Hz}), 2.09(\mathrm{~s}, 3 \mathrm{H}), 1.87(\mathrm{~m}, 2 \mathrm{H}), 1.76(\mathrm{~m}, 2 \mathrm{H}), 1.65(\mathrm{~m}, 2 \mathrm{H})$, $1.44(\mathrm{~m}, 2 \mathrm{H}), 0.94(\mathrm{t}, 3 \mathrm{H}, \mathrm{J}=7.2 \mathrm{~Hz}) ;{ }^{13} \mathrm{C}$ NMR $\left(500 \mathrm{MHz}, \mathrm{CDCl}_{3}\right) \delta 158.0,142.5,127.6$, 118.9, 99.2, 58.7, 51.2, 46.9, 33.7, 24.2, 21.0, 20.9, 20.1, 16.0, 14.1; IR (neat) $\mathrm{cm}^{-1} 2930$, 2858, 1614, 1579, 1466, 1424, 1397, 1322, 1230, 1183, 1118, 996, 759, 667; HRMS m/z calc. for $\mathrm{C}_{15} \mathrm{H}_{24} \mathrm{ON}_{2}\left(\mathrm{M}^{+}\right)$248.1883, found 248.18930.

\section{Preparation of compound $1 \mathrm{e}^{3}$}

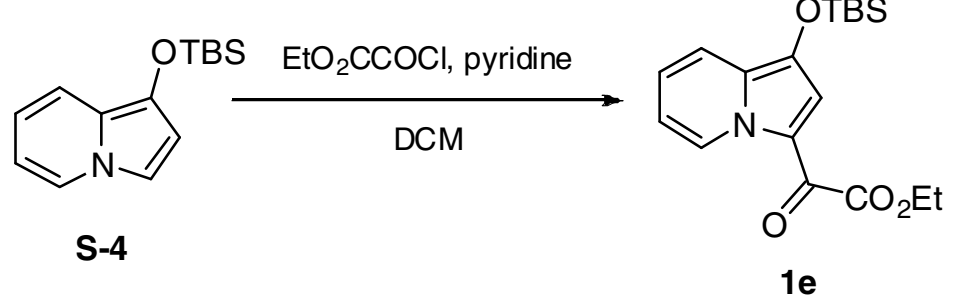

A solution of pyridine $(48 \mathrm{mg}, 0.6 \mathrm{mmol})$ in dry dichloromethane $(1.5 \mathrm{~mL})$ was added dropwise to a stirred solution of ethyl chlorooxoacetate $(75 \mathrm{mg}, 0.55 \mathrm{mmol})$ in dry dichloromethane $(1.5 \mathrm{~mL})$ at $-78^{\circ} \mathrm{C}$ under nitrogen. A solution of the 1-((t-butyldimethyl)silanyloxy)indolizine $\quad \mathrm{S}^{-4} \mathbf{}^{7}(123 \mathrm{mg}, \quad 0.5 \mathrm{mmol})$ in dry dichloromethane $(1.5 \mathrm{~mL})$ was added dropwise to it and the solution allowed to warm slowly to room temperature. After being stirred for $48 \mathrm{~h}$, the solution was washed with dilute hydrochloric acid, water and brine, dried $\left(\mathrm{MgSO}_{4}\right)$ and evaporated. The residue was purified by column chromatography (silica gel, diethyl ether/hexane 1:3) to give a $108 \mathrm{mg}$

7 1-((t-butyldimethyl)silanyloxy)indolizine was prepared via published procedure. Seregin, I. V.; Gevorgyan, V. J. Am. Chem. Soc. 2006, 128, 12050-12051. 
$(62 \%, 0.31 \mathrm{mmol})$ of the title compound 1e. ${ }^{1} \mathrm{H}$ NMR $\left(400 \mathrm{MHz}, \mathrm{CDCl}_{3}\right): 9.96(\mathrm{~d}, 1 \mathrm{H}, \mathrm{J}=$ $7.2 \mathrm{~Hz}), 7.59(\mathrm{~d}, 1 \mathrm{H}, \mathrm{J}=8.8 \mathrm{~Hz}), 7.36(\mathrm{~s}, 1 \mathrm{H}), 7.27(\mathrm{~m}, 1 \mathrm{H}), 7.00\left(\mathrm{td}, 1 \mathrm{H}, \mathrm{J}_{1}=6.8 \mathrm{~Hz}, \mathrm{~J}_{2}=\right.$ $1.2 \mathrm{~Hz}), 4.43(\mathrm{q}, 2 \mathrm{H}, \mathrm{J}=7.2 \mathrm{~Hz}), 1.45(\mathrm{t}, 3 \mathrm{H}, \mathrm{J}=7.2 \mathrm{~Hz}), 1.05(\mathrm{~s}, 9 \mathrm{H}), 0.27(\mathrm{~s}, 6 \mathrm{H}) ;{ }^{13} \mathrm{C}$ NMR $\left(500 \mathrm{MHz}, \mathrm{CDCl}_{3}\right) \delta 168.7,163.8,135.8,134.0,129.2,125.4,116.6,116.2,115.6$, 112.4, 61.9, 25.7, 18.2, 14.2, -4.7; IR (neat) $\mathrm{cm}^{-1} 1723,1594,1555,1523,1477,1258$, $1224,1166,1117,1083,1025,931,870,832,755,594 ;$ HRMS $m / z$ calc. for $\mathrm{C}_{18} \mathrm{H}_{25} \mathrm{O}_{4} \mathrm{NSi}$ $\left(\mathrm{M}+\mathrm{H}^{+}\right) 348.1626$, found 348.16357 .

\section{Preparation of compound $1 \mathrm{f}^{8}$}
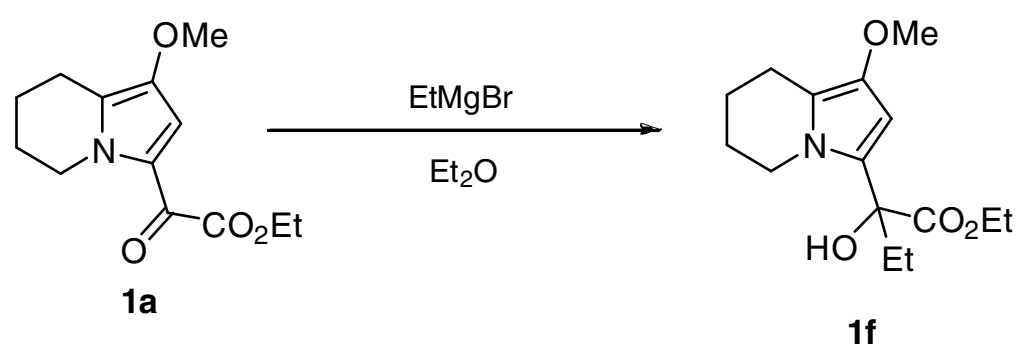

To a solution of pyrrole $1 \mathbf{a}(50 \mathrm{mg}, 0.2 \mathrm{mmol})$ in $2 \mathrm{~mL}$ of dry diethyl ether at $-78^{\circ} \mathrm{C}$, under nitrogen, was added ethyl magnesiumchloride ( $3.0 \mathrm{M}$ solution in $\mathrm{Et}_{2} \mathrm{O}, 0.07 \mathrm{~mL}, 1.05$ eq.) dropwise. After the addition, the cooling bath was removed, and the reaction was stirred for $12 \mathrm{~h}$ while allowing to slowly warm up to room temperature. The reaction was quenched with aqueous ammonium chloride $(10 \mathrm{~mL}) .20 \mathrm{~mL}$ of ethyl acetate was then added and the mixture transferred to a separatory funnel. The layers were separated and the organic layer was washed with water, brine, and dried over $\mathrm{Na}_{2} \mathrm{SO}_{4}$. After filtration, the solvents were evaporated and the product is purified by column chromatography (silica gel, ethyl acetate/hexane 1:1) to give a $48 \mathrm{mg}$ (85\% yield) of the title compound 1f. ${ }^{1} \mathrm{H}$ NMR (500 $\left.\mathrm{MHz}, \mathrm{CDCl}_{3}\right): 5.96(\mathrm{~s}, 1 \mathrm{H}), 4.36-4.32(\mathrm{~m}, 1 \mathrm{H}), 4.21-4.18(\mathrm{~m}, 1 \mathrm{H}), 4.08-4.06(\mathrm{~m}, 1 \mathrm{H}), 3.73$ $(\mathrm{s}, 3 \mathrm{H}), 3.56(\mathrm{~m}, 2 \mathrm{H}), 2.67(\mathrm{~m}, 2 \mathrm{H}), 2.26-2.22(\mathrm{~m}, 1 \mathrm{H}), 2.14-2.10(\mathrm{~m}, 1 \mathrm{H}), 1.90-1.67(\mathrm{~m}$, $4 \mathrm{H}), 1.27(\mathrm{t}, 3 \mathrm{H}, \mathrm{J}=7.0 \mathrm{~Hz}), 0.94(\mathrm{t}, 3 \mathrm{H}, \mathrm{J}=7.5 \mathrm{~Hz}) ;{ }^{13} \mathrm{C} \mathrm{NMR}\left(500 \mathrm{MHz}, \mathrm{CDCl}_{3}\right) \delta 175.4$, $141.7,125.9,115.0,95.9,75.0,62.4,58.7,43.9,31.2,23.9,20.7,20.4,14.2,7.5$; IR (neat) $\mathrm{cm}^{-1} 2940,1736,1722,1634,1626,1372,1326,1234,1131,1118,1020 ;$ HRMS $m / z$ calc. for $\mathrm{C}_{15} \mathrm{H}_{23} \mathrm{O}_{4} \mathrm{~N}\left(\mathrm{M}+\mathrm{H}^{+}\right) 282.1700$, found 282.16983.

\footnotetext{
${ }^{8}$ Dettwiler, J. E.; Lubell, W. D. J. Org. Chem. 2003, 68, 177-179.
} 


\section{Preparation of compound $1 \mathrm{~g}$}<smiles>CCOC(=O)c1c(OC)ccn1C</smiles>

$1 \mathrm{~g}$ was prepared by the general procedure from sarcosine methyl ester hydrochloride $(2.1 \mathrm{~g}$, $15 \mathrm{mmol}$ ). Acylation of 3-methoxy-1-methylpyrrole occurred at the $o$-position to give compound 1g. Purification by column chromatography on silica gel (ethyl acetate/hexane 1:1) gave $0.58 \mathrm{~g}$ of $\mathbf{1 g}$ (45\% yield). ${ }^{1} \mathrm{H} \mathrm{NMR}\left(400 \mathrm{MHz}, \mathrm{CDCl}_{3}\right): 6.76(\mathrm{~d}, 1 \mathrm{H}, \mathrm{J}=2.8 \mathrm{~Hz})$, $5.73(\mathrm{~d}, 1 \mathrm{H}, \mathrm{J}=2.8 \mathrm{~Hz}), 4.34(\mathrm{q}, 2 \mathrm{H}, \mathrm{J}=7.2 \mathrm{~Hz}), 3.86(\mathrm{~s}, 3 \mathrm{H}), 3.78(\mathrm{~s}, 3 \mathrm{H}), 1.35$ (t, 3H, J = $7.2 \mathrm{~Hz}) ;{ }^{13} \mathrm{C}$ NMR $\left(500 \mathrm{MHz}, \mathrm{CDCl}_{3}\right) \delta 174.2,165.5,158.7,132.6,114.0,93.4,93.4,61.4$, 58.1, 37.9, 14.0; IR (neat) $\mathrm{cm}^{-1} 1738,1614,1527,1454,1432,1418,1370,1357,1286$, $1247,1207,1104,1017,978,754,709 ;$ HRMS $m / z$ calc. for $\mathrm{C}_{10} \mathrm{H}_{13} \mathrm{O}_{4} \mathrm{~N}\left(\mathrm{M}+\mathrm{H}^{+}\right)$ 212.0917, found 212.09070.

\section{Preparation of compound $1 \mathrm{~h}$}<smiles>CCOC(=O)c1cc(OC)c(C)n1C</smiles>

1h was prepared by the general procedure from $N$-methyl-L-alanine methyl ester hydrochloride $(0.46 \mathrm{~g}, 3.0 \mathrm{mmol}){ }^{9}$ Purified by column chromatography on silica gel, ethyl acetate/hexane $1: 1$, to give $43 \mathrm{mg}$ of $\mathbf{1 h}$ (35\% yield). ${ }^{1} \mathrm{H} \mathrm{NMR}\left(400 \mathrm{MHz}, \mathrm{CDCl}_{3}\right.$ ): $6.81(\mathrm{~s}, 1 \mathrm{H}), 4.36(\mathrm{q}, 2 \mathrm{H}, \mathrm{J}=7.2 \mathrm{~Hz}), 3.88(\mathrm{~s}, 3 \mathrm{H}), 3.75$ (s, 3H), 2.19 (s, 3H), 1.40 (t, 3H, J $=7.2 \mathrm{~Hz}) ;{ }^{13} \mathrm{C} \mathrm{NMR}\left(500 \mathrm{MHz}, \mathrm{CDCl}_{3}\right) \delta 171.9,164.1,146.0,130.5,122.8,106.8,61.8$, 58.0, 33.4, 14.1, 8.57; IR (neat) $\mathrm{cm}^{-1} 1737,1729,1633,1615,1563,1557,1418,1356$, $1279,1202,1171,1155,1133,1021,938,756$; HRMS $m / z$ calc. for $\mathrm{C}_{11} \mathrm{H}_{15} \mathrm{O}_{4} \mathrm{~N}\left(\mathrm{M}+\mathrm{H}^{+}\right)$ 226.1074, found 226.10757 .

\footnotetext{
9 2-(Methylamino)propanoic acid was prepared via published procedure. Cheung, S. T.; Benoiton, N. L. Can. J. Chem. 1977, 55, 906-910.
} 


\section{Preparation of compound $1 i^{10}$}

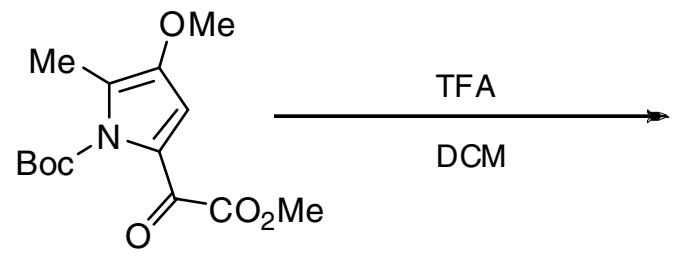

1j

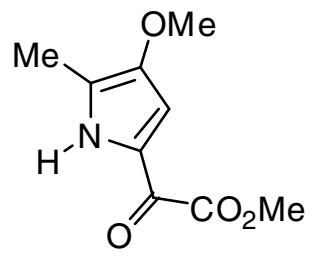

$1 \mathbf{i}$

TFA (5 ml) was added dropwise to a solution of the pyrrole $\mathbf{1 j}(50 \mathrm{mg}, 0.17 \mathrm{mmol})$ in dichloromethane $(5 \mathrm{ml})$ at room temperature. After $2 \mathrm{~h}$ the solution was evaporated and The residue was purified by column chromatography (silica gel, ethyl acetate/hexane 1:1) to give a $20 \mathrm{mg}$ (60\% yield) of the title compound 1i. ${ }^{1} \mathrm{H} \mathrm{NMR}\left(400 \mathrm{MHz}, \mathrm{CDCl}_{3}\right)$ : 9.95 (s, 1H) $6.99(\mathrm{~s}, 1 \mathrm{H}), 3.92(\mathrm{~s}, 3 \mathrm{H}), 3.78(\mathrm{~s}, 3 \mathrm{H}), 2.28(\mathrm{~s}, 3 \mathrm{H}) ;{ }^{13} \mathrm{C} \mathrm{NMR}\left(500 \mathrm{MHz}, \mathrm{CDCl}_{3}\right) \delta$ $168.8,163.1,147.7,128.9,123.7,106.6,58.1,52.7,10.2$; IR (neat) $\mathrm{cm}^{-1} 3233,1734,1627$, 1568, 1542, 1487, 1421, 1390, 1307, 1256, 1174, 1153, 1029, 824, 761; HRMS m/z calc. for $\mathrm{C}_{9} \mathrm{H}_{11} \mathrm{O}_{4} \mathrm{~N}\left(\mathrm{M}+\mathrm{H}^{+}\right)$198.0761, found 198.07550.

\section{Preparation of compound $\mathbf{1 j}$}<smiles>COC(=O)c1cc(OC)c(C)n1C(=O)OCc1ccccc1</smiles>

1j was prepared by the general procedure from D-alanine methyl ester hydrochloride (1.4 $\mathrm{g}, 10 \mathrm{mmol}$ ). Boc protection ${ }^{9}$ on alkoxypyrrolinone $\mathbf{S - 3}$ was done before the DIBAL reduction. Purification by column chromatography on silica gel, diethyl ether/hexane 1:1, gave $0.5 \mathrm{~g}$ of $\mathbf{1 j}$ (28\% yield). ${ }^{1} \mathrm{H}$ NMR (400 MHz, $\left.\mathrm{CDCl}_{3}\right): 6.70(\mathrm{~s}, 1 \mathrm{H}), 3.90(\mathrm{~s}, 3 \mathrm{H}), 3.78$ $(\mathrm{s}, 3 \mathrm{H}), 2.30(\mathrm{~s}, 3 \mathrm{H}), 1.59(\mathrm{~s}, 9 \mathrm{H}) ;{ }^{13} \mathrm{C} \mathrm{NMR}\left(500 \mathrm{MHz}, \mathrm{CDCl}_{3}\right) \delta 171.8,162.8,149.4$, 146.2, 126.3, 125.8, 110.7, 86.0, 58.5, 52.8, 27.5, 10.0; IR (neat) $\mathrm{cm}^{-1} 1761,1734,1652$, $1476,1415,1371,1324,1291,1260,1159,1120$; HRMS $m / z$ calc. for $\mathrm{C}_{14} \mathrm{H}_{19} \mathrm{O}_{6} \mathrm{~N}(\mathrm{M}+$ $\mathrm{Na}^{+}$) 320.1105, found 320.11099.

\section{Preparation of compound 1k}

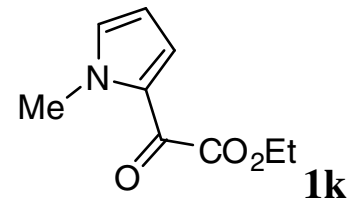

1k was prepared by the acylation ${ }^{3}$ of 1 -methylpyrrole with ethyl chlorooxoacetate $(0.23 \mathrm{~g}$, $2.9 \mathrm{mmol})$. Purified by column chromatography on silica gel, diethyl ether/hexane $1: 1$, to

${ }^{10}$ Gilchrist, T. L.; Lemos, A.; Ottaway, C. J. J. Chem. Soc., Perkin Trans. 1 1997, 3005-3008. 
give $0.43 \mathrm{~g}$ of $1 \mathbf{k}\left(83 \%\right.$ yield). ${ }^{1} \mathrm{H}$ NMR $\left(400 \mathrm{MHz}, \mathrm{CDCl}_{3}\right): 7.28,(\mathrm{~m}, 1 \mathrm{H}), 6.98(\mathrm{~d}, 1 \mathrm{H}, \mathrm{J}=$ $2.0 \mathrm{~Hz}), 6.22\left(\mathrm{q}, 1 \mathrm{H}, \mathrm{J}_{1}=4.0 \mathrm{~Hz}, \mathrm{~J}_{2}=2.4 \mathrm{~Hz}\right), 4.40(\mathrm{q}, 2 \mathrm{H}, \mathrm{J}=7.2 \mathrm{~Hz}), 3.99(\mathrm{~s}, 3 \mathrm{H}), 1.41(\mathrm{t}$, $3 \mathrm{H}, \mathrm{J}=7.2 \mathrm{~Hz}) ;{ }^{13} \mathrm{C} \mathrm{NMR}\left(500 \mathrm{MHz}, \mathrm{CDCl}_{3}\right) \delta 174.4,163.5,134.1,127.5,124.8,109.7$, 62.0, 37.6, 14.1; IR (neat) $\mathrm{cm}^{-1} 1735,1643,1405,1366,1335,1267,1237,1200,1062$, $1040,1014,961,747$; HRMS $m / z$ calc. for $\mathrm{C}_{9} \mathrm{H}_{11} \mathrm{O}_{3} \mathrm{~N}\left(\mathrm{M}^{+}\right)$181.0733, found 181.07353.

\section{Preparation of compound $11^{11}$}<smiles>CCOC(=O)c1c(Br)ccn1C</smiles>

S-5

\section{1. $2.5 \%\left(\mathrm{Ph}_{3} \mathrm{P}\right)_{4} \mathrm{Pd}$}

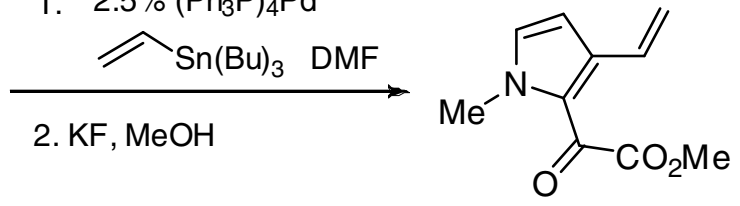

11

11

Tetrakis(triphenylphosphane) palladium(0) $(29 \mathrm{mg}, 25 \mu \mathrm{mol})$ in dry DMF $(7 \mathrm{~mL})$ was added to a solution of bromopyrrole $\mathbf{S - 5} 5^{12}(260 \mathrm{mg}, 1.00 \mathrm{mmol})$ and ethenyl(tributyl)tin $(0.379 \mathrm{~mL}, 1.30 \mathrm{mmol})$ in DMF ( $3 \mathrm{~mL}$ ). The resulting mixture was stirred for $23 \mathrm{~h}$ under Ar at $100{ }^{\circ} \mathrm{C}$, cooled and evaporated in vacuo. The residue was dissolved in a saturated solution of $\mathrm{KF}$ in $\mathrm{MeOH}$ and stirred for $2 \mathrm{~h}$. The mixture was evaporated in vacuo and the product was purified by column chromatography (silica gel, diethyl ether/hexane 1:3) to give a $180 \mathrm{mg}(87 \%, 0.87 \mathrm{mmol})$ of the title compound $11 .{ }^{1} \mathrm{H} \mathrm{NMR}\left(400 \mathrm{MHz}, \mathrm{CDCl}_{3}\right)$ : 6.87, (d, $1 \mathrm{H}, \mathrm{J}=3.0 \mathrm{~Hz}), 6.62\left(\mathrm{dd}, 1 \mathrm{H}, \mathrm{J}_{1}=21.0 \mathrm{~Hz}, \mathrm{~J}_{2}=13.5 \mathrm{~Hz}\right) 6.34(\mathrm{~d}, 1 \mathrm{H}, \mathrm{J}=3.0 \mathrm{~Hz})$, $5.60(\mathrm{~d}, 1 \mathrm{H}, \mathrm{J}=21.0 \mathrm{~Hz}), 5.28(\mathrm{~d}, 1 \mathrm{H}, \mathrm{J}=13.5 \mathrm{~Hz}), 3.92(\mathrm{~s}, 3 \mathrm{H}) ;{ }^{13} \mathrm{C} \mathrm{NMR}(500 \mathrm{MHz}$, $\left.\mathrm{CDCl}_{3}\right) \delta 176.4,165.5,135.2,132.9,127.7,123.8,117.4,107.6,52.7,38.0$; IR (neat) $\mathrm{cm}^{-1}$ 1737, 1621, 1513, 1427, 1403, 1356, 1286, 1237, 1202, 1041, 1008, 993, 783, 770, 603, 500; HRMS $m / z$ calc. for $\mathrm{C}_{10} \mathrm{H}_{11} \mathrm{O}_{3} \mathrm{~N}\left(\mathrm{M}^{+}\right)$193.0733, found 193.07257.

\section{Preparation of compound $1 \mathrm{~m}^{11}$}<smiles>CCOC(=O)c1cc(Br)cn1C</smiles>

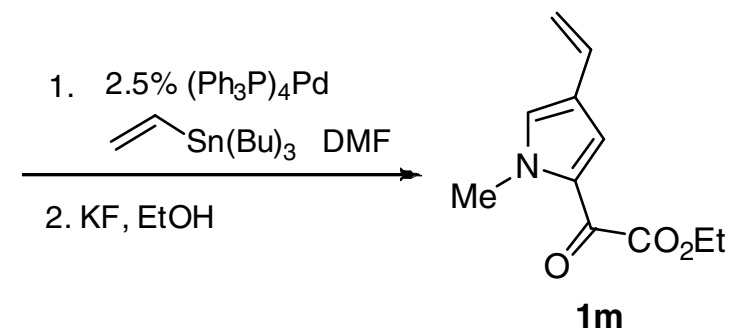

Tetrakis(triphenylphosphane) palladium(0) $(16 \mathrm{mg}, 14 \mu \mathrm{mol})$ in dry DMF $(4 \mathrm{~mL})$ was added to a solution of bromopyrrole $\mathbf{S - 6}{ }^{13}$ (144 $\mathrm{mg}, 0.55 \mathrm{mmol}$ ) and ethenyl(tributyl)tin

\footnotetext{
${ }^{11}$ Østby, O. B.; Dalhus, B.; Gundersen, L.-L.; Rise, F.; Bast, A.; Haenen, G. R. M. M. Eur. J. Org. Chem. 2000, 3763-3770.

12 Compound S-5 was prepared by the acylation of 3-bromo- $N$-methylpyrrole with ethyl chlorooxoacetate (Ref. 3). 3-bromo- $N$-methylpyrrole was prepared via published procedure (Dvornikova, E.; Kamieska-Trela, K. Synlett 2002, 1152-1154.)

${ }^{13}$ Compound S-6 was prepared by the acylation of 3-bromo- $N$-methylpyrrole with ethyl chlorooxoacetate (Ref. 3).
} 
(0.21 mL, $0.72 \mathrm{mmol}$ ) in DMF (2 mL). The resulting mixture was stirred for $23 \mathrm{~h}$ under Ar at $100{ }^{\circ} \mathrm{C}$, cooled and evaporated in vacuo. The residue was dissolved in a saturated solution of $\mathrm{KF}$ in $\mathrm{EtOH}$ and stirred for $2 \mathrm{~h}$. The mixture was evaporated in vacuo and the product was purified by column chromatography (silica gel, diethyl ether/hexane 1:3) to give a $78 \mathrm{mg}(68 \%, 0.37 \mathrm{mmol})$ of the title compound $\mathbf{1 m} .{ }^{1} \mathrm{H}$ NMR $\left(400 \mathrm{MHz}, \mathrm{CDCl}_{3}\right)$ : 7.32, (d, 1H, J = 2.0 Hz), $7.02(\mathrm{~s}, 1 \mathrm{H}), 6.49\left(\mathrm{dd}, 1 \mathrm{H}, \mathrm{J}_{1}=17.5 \mathrm{~Hz}, \mathrm{~J}_{2}=10.5 \mathrm{~Hz}\right), 5.48(\mathrm{~d}, 1 \mathrm{H}$, $\mathrm{J}=17.5 \mathrm{~Hz}), 5.08(\mathrm{~d}, 1 \mathrm{H}, \mathrm{J}=10.5 \mathrm{~Hz}), 4.40(\mathrm{q}, 2 \mathrm{H}, \mathrm{J}=7.0 \mathrm{~Hz}), 3.95(\mathrm{~s}, 3 \mathrm{H}), 1.42(\mathrm{t}, 3 \mathrm{H}, \mathrm{J}$ $=7.0 \mathrm{~Hz}) ;{ }^{13} \mathrm{C} \mathrm{NMR}\left(500 \mathrm{MHz}, \mathrm{CDCl}_{3}\right) \delta 174.5,163.3,132.1,128.2,127.8,123.9,121.1$, 112.3, 62.2, 37.8, 14.1; IR (neat) $\mathrm{cm}^{-1} 1734,1646,1445,1405,1372,1363,1281,1226$, 1185, 1150, 1018; HRMS $m / z$ calc. for $\mathrm{C}_{11} \mathrm{H}_{13} \mathrm{O}_{3} \mathrm{~N}\left(\mathrm{M}+\mathrm{H}^{+}\right)$208.0968, found 208.09668.

\section{General Procedure for the Heterogeneous Hydrogenation}

The hydrogenation reactions at 10 atm were carried out in open vials in a pressure vessel (4765 $600 \mathrm{ml}$ vessel with split ring closure and 4316 gage block assembly, Parr instrument company) equipped with glass liner and PTFE cover. The reactor was magnetically stirred. The standard reaction conditions were catalyst (w/w with substrate, $2.5 \mathrm{~mol} \%$ ), 0.20 mmol reactant, $1 \mathrm{ml}$ solvent $(\mathrm{MeOH}$ or $\mathrm{EtOH})$, a pressure of $10 \mathrm{~atm}$, room temperature, and a reaction time of $24 \mathrm{~h}$. The catalyst was filtered off through a Celite pad and the solvent was removed under reduced pressure. The product ratio was determined by ${ }^{1} \mathrm{H}$ NMR of the crude mixture. The residue was purified by column chromatography on silica gel (ethyl acetate, ethyl acetate/methanol 9:1, ethyl acetate/methanol/triethylamine 9:1:0.2) to give the pyrrolidine product.

Some hydrogenations reactions could be done under 1 atm of hydrogen, such as the hydrogenations of compound 1a and 1k (Scheme 3 and 4 in the paper). Hydrogenations of other compounds 1 under 1 atm of hydrogen were not tested. The hydrogenation reactions

at 1 atm were carried out in closed vials charged with $\mathrm{H}_{2}$ via a hydrogen balloon and followed the same procedure as in the 10 atm reactions above. 


\section{Tabulated Spectral Data of Hydrogenation Products}<smiles>CCOC(O)C1CC(OC)[C@@H]2CCCCN12</smiles>

(Purified by column chromatography on silica gel, ethyl acetate, 92\%) ${ }^{1} \mathrm{H}$ NMR $(400 \mathrm{MHz}$, $\left.\mathrm{CDCl}_{3}\right): 4.30(\mathrm{~d}, 1 \mathrm{H}, \mathrm{J}=2.8 \mathrm{~Hz}), 4.25(\mathrm{q}, 2 \mathrm{H}, \mathrm{J}=7.2 \mathrm{~Hz}), 3.56(\mathrm{t}, 1 \mathrm{H}, \mathrm{J}=4.0 \mathrm{~Hz}), 3.29$ (s, $3 \mathrm{H}), 3.12(\mathrm{~m}, 1 \mathrm{H}), 2.86(\mathrm{~m}, 1 \mathrm{H}), 2.18(\mathrm{~m}, 1 \mathrm{H}), 2.05(\mathrm{~m}, 1 \mathrm{H}), 1.82(\mathrm{~m}, 3 \mathrm{H}), 1.66(\mathrm{~m}, 2 \mathrm{H})$, $1.62(\mathrm{~m}, 1 \mathrm{H}), 1.51(\mathrm{~m}, 1 \mathrm{H}), 1.30(\mathrm{t}, 3 \mathrm{H}, \mathrm{J}=7.2 \mathrm{~Hz}), 1.21(\mathrm{~m}, 1 \mathrm{H}) ;{ }^{13} \mathrm{C}$ NMR $(500 \mathrm{MHz}$, $\left.\mathrm{CDCl}_{3}\right) \delta 172.3,80.0,68.6,68.1,65.3,60.9,56.5,50.8,28.4,25.3,25.2,24.0,14.2$; IR (neat) $\mathrm{cm}^{-1} 3394,2938,1738,1608,1450,1373,1295,1269,1202,1144,1107,1075,1025$, 913, 733, 693; HRMS $m / z$ calc. for $\mathrm{C}_{13} \mathrm{H}_{23} \mathrm{O}_{4} \mathrm{~N}\left(\mathrm{M}+\mathrm{H}^{+}\right)$258.1700, found 258.16937.<smiles>CO[C@H]1C[C@H](C([14CH3])O)N2CCCC[C@H]12</smiles>

In this case, a second portion of catalyst (w/w with the substrate) was added to drive the reaction to completion. (Purified by column chromatography on silica gel, ethyl acetate, 90\%) ${ }^{1} \mathrm{H}$ NMR (500 MHz, $\left.\mathrm{CDCl}_{3}\right): 3.88(\mathrm{~m}, 1 \mathrm{H}), 3.57(\mathrm{t}, 1 \mathrm{H}, \mathrm{J}=4.5 \mathrm{~Hz}), 3.27(\mathrm{~s}, 3 \mathrm{H})$, $3.12(\mathrm{~d}, 1 \mathrm{H}, \mathrm{J}=10 \mathrm{~Hz}), 2.26-2.23(\mathrm{~m}, 1 \mathrm{H}), 2.11-2.07(\mathrm{~m}, 1 \mathrm{H}), 1.91-1.77(\mathrm{~m}, 4 \mathrm{H})$, $1.68-1.59(\mathrm{~m}, 3 \mathrm{H}), 1.49-1.46(\mathrm{~m}, 1 \mathrm{H}), 1.23-1.19(\mathrm{~m}, 1 \mathrm{H}), 1.10(\mathrm{~d}, 3 \mathrm{H}, \mathrm{J}=7.2 \mathrm{~Hz}) ;{ }^{13} \mathrm{C}$ NMR $\left(500 \mathrm{MHz}, \mathrm{CDCl}_{3}\right) \delta 80.3,69.1,68.6,63.0,56.5,51.3,27.7,25.6,25.5,24.4,18.6$; IR (neat) $\mathrm{cm}^{-1} 2934,2856,2819,2790,1452,1442,1369,1346,1290,1262,1205,1144$, 1114, 1090, 1076, 1062, 1054; HRMS $m / z$ calc. for $\mathrm{C}_{11} \mathrm{H}_{21} \mathrm{O}_{2} \mathrm{~N}\left(\mathrm{M}+\mathrm{H}^{+}\right) 200.1645$, found 200.16389.

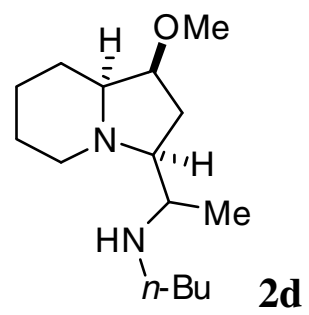

In this case, a second portion of catalyst (w/w with the substrate) was added to drive the reaction to completion. (Purified by column chromatography on silica gel, ethyl acetate/methanol/triethylamine 9:1:0.2, 75\%) ${ }^{1} \mathrm{H}$ NMR (400 MHz, $\left.\mathrm{CDCl}_{3}\right): 3.59-3.57$ (m, $1 \mathrm{H}), 3.28(\mathrm{~s}, 3 \mathrm{H}), 3.15(\mathrm{~m}, 1 \mathrm{H}, \mathrm{J}=13.5 \mathrm{~Hz}), 2.93-2.87(\mathrm{~m}, 1 \mathrm{H}), 2.73-2.67(\mathrm{~m}, 1 \mathrm{H})$, 2.59-2.52 (m, 1H), 2.32-2.27 (m, 1H), 1.95-1.87 (m, 2H), 1.84-1.78 (m, 2H), 1.67-1.44 (m, 
$8 \mathrm{H}), 1.39-1.30(\mathrm{~m}, 2 \mathrm{H}), 1.26-1.14(\mathrm{~m}, 1 \mathrm{H}) 1.03(\mathrm{~d}, 3 \mathrm{H}, \mathrm{J}=6.8 \mathrm{~Hz}), 0.92(\mathrm{t}, 3 \mathrm{H}, \mathrm{J}=7.2 \mathrm{~Hz})$;

${ }^{13} \mathrm{C} \mathrm{NMR}\left(500 \mathrm{MHz}, \mathrm{CDCl}_{3}\right) \delta 80.6,69.9,67.7,56.6,54.1,53.1,47.5,32.4,30.6,25.6$, 25.4, 24.5, 20.6, 14.9, 14.0; IR (neat) 2954, 2931, 2872, 2856, 2816, 2794, 2758, 2718, 1452, 1442, 1369, 1352, 1264, 1204, 1198, 1146, 1117, 1085, $1062 \mathrm{~cm}^{-1}$; HRMS m/z calc. for $\mathrm{C}_{15} \mathrm{H}_{30} \mathrm{ON}_{2}\left(\mathrm{M}+\mathrm{H}^{+}\right) 255.2431$, found 255.24188

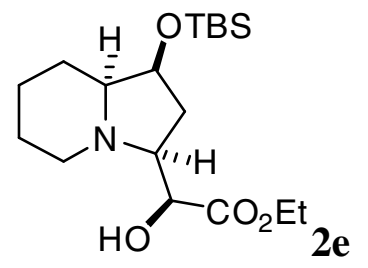

(Purified by column chromatography on silica gel, ethyl acetate/hexane $1: 1,91 \%){ }^{1} \mathrm{H}$ NMR (400 MHz, $\left.\mathrm{CDCl}_{3}\right)$ : 4.31-4.14 (m, 3H), 4.04-4.02 (m, 1H), 3.13-3.11 (m, 1H), 2.90-2.85 (m, 1H), 2.14-2.05 (m, 2H), 1.98-1.91 (m, 1H), 1.83-1.80 (m, 1H), 1.69-1.44 (m, $6 \mathrm{H}), 1.28(\mathrm{t}, 3 \mathrm{H}, \mathrm{J}=7.2 \mathrm{~Hz}), 0.90(\mathrm{~s}, 9 \mathrm{H}), 0.08-0.05(\mathrm{~m}, 6 \mathrm{H}) ;{ }^{13} \mathrm{C} \mathrm{NMR}\left(500 \mathrm{MHz}, \mathrm{CDCl}_{3}\right)$ $\delta 172.6,71.8,68.9,68.4,64.9,60.6,50.5,33.6,26.0,25.8,25.4,23.9,18.2,14.2,-4.8,-5.0$; IR (neat) $\mathrm{cm}^{-1} 3441,2934,2855,1751,1657,1464,1387,1256,1179,1143,1094,1024$, 949, 836, 775, 708; HRMS $m / z$ calc. for $\mathrm{C}_{18} \mathrm{H}_{35} \mathrm{O}_{4} \mathrm{NSi}\left(\mathrm{M}+\mathrm{H}^{+}\right)$358.2408, found 358.24005 .

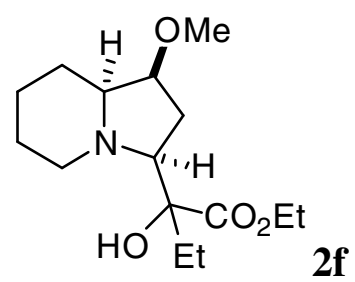

(Purified by column chromatography on silica gel, ethyl acetate, 91\%) ${ }^{1} \mathrm{H}$ NMR $(500 \mathrm{MHz}$, $\left.\mathrm{CDCl}_{3}\right): 4.24(\mathrm{dd}, 2 \mathrm{H}, \mathrm{J}=5.6 \mathrm{~Hz}), 3.96(\mathrm{~m}, 1 \mathrm{H}), 3.52(\mathrm{t}, 1 \mathrm{H}, \mathrm{J}=3.5 \mathrm{~Hz}), 3.29(\mathrm{~s}, 3 \mathrm{H}), 3.00$ $\left(\mathrm{dd}, 1 \mathrm{H}, \mathrm{J}_{1}=4.0 \mathrm{~Hz}, \mathrm{~J}_{2}=9.5 \mathrm{~Hz}\right), 2.24-2.19(\mathrm{~m}, 2 \mathrm{H}), 1.92-1.90(\mathrm{~m}, 1 \mathrm{H}), 1.87-1.83(\mathrm{~m}, 1 \mathrm{H})$, $1.80-1.75(\mathrm{~m}, 3 \mathrm{H}), 1.65-1.61(\mathrm{~m}, 3 \mathrm{H}), 1.57-1.52(\mathrm{~m}, 2 \mathrm{H}), 1.29(\mathrm{t}, 3 \mathrm{H}, \mathrm{J}=7.0 \mathrm{~Hz})$, $1.26-1.15(\mathrm{~m}, 1 \mathrm{H}), 0.90(\mathrm{t}, 3 \mathrm{H}, \mathrm{J}=7.0 \mathrm{~Hz}) ;{ }^{13} \mathrm{C} \mathrm{NMR}\left(500 \mathrm{MHz}, \mathrm{CDCl}_{3}\right) \delta 174.7,80.8$, $80.7,70.1,67.7,60.8,56.5,55.8,31.3,31.1,26.2,26.0,24.4,14.4,8.5$; IR (neat) cm $\mathrm{cm}^{-1} 2932$, 2854, 1727, 1463, 1435, 1371, 1298, 1235, 1206, 1154, 1139, 1119, 1111, 1096, 1023; HRMS $m / z$ calc. for $\mathrm{C}_{15} \mathrm{H}_{27} \mathrm{O}_{4} \mathrm{~N}\left(\mathrm{M}+\mathrm{H}^{+}\right)$286.2013, found 286.20132.<smiles>CCOC(O)C(O)C1C(OC)CCN1C</smiles>

In this case, a second portion of catalyst (w/w with the substrate) was added to drive the reaction to completion. (Purified by column chromatography on silica gel, ethyl acetate, 
91\%) ${ }^{1} \mathrm{H}$ NMR $\left(400 \mathrm{MHz}, \mathrm{CDCl}_{3}\right): 4.33(\mathrm{~d}, 1 \mathrm{H}, \mathrm{J}=4.0 \mathrm{~Hz}), 4.27(\mathrm{q}, 2 \mathrm{H}, \mathrm{J}=7.0 \mathrm{~Hz})$, 3.98-3.94 (m, 1H), 3.26-3.22 (m, 4H), 2.67-2.65 (m, 1H), $2.40(\mathrm{~s}, 3 \mathrm{H}), 2.22-2.18(\mathrm{~m}, 1 \mathrm{H})$, 2.09-2.06 (m, 1H), 1.84-1.82 (m, 1H), $1.32(\mathrm{t}, 3 \mathrm{H}, \mathrm{J}=7.0 \mathrm{~Hz}) ;{ }^{13} \mathrm{C} \mathrm{NMR}\left(500 \mathrm{MHz}, \mathrm{CDCl}_{3}\right)$ $\delta 173.3,82.8,70.0,69.3,60.9,56.6,54.2,39.9,30.1,14.4$; IR (neat) $\mathrm{cm}^{-1} 3472,2976,2939$, $1743,1454,1280,1235,1200,1143,1109,1078,1030$; HRMS $m / z$ calc. for $\mathrm{C}_{10} \mathrm{H}_{19} \mathrm{O}_{4} \mathrm{~N}$ $\left(\mathrm{M}+\mathrm{H}^{+}\right)$218.1387, found 218.13820.

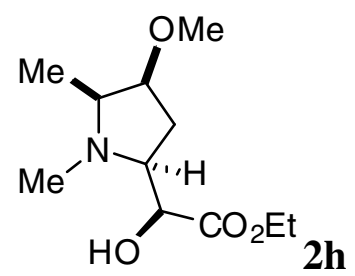

(Purified by column chromatography on silica gel, ethyl acetate, 90\%) ${ }^{1} \mathrm{H}$ NMR $(500 \mathrm{MHz}$, $\left.\mathrm{CDCl}_{3}\right)$ : $4.25(\mathrm{~d}, 1 \mathrm{H}, \mathrm{J}=2.5 \mathrm{~Hz}), 4.24-4.20(\mathrm{~m}, 2 \mathrm{H}), 3.53-3.51(\mathrm{~m}, 1 \mathrm{H}), 3.29(\mathrm{~s}, 3 \mathrm{H})$, 2.93-2.90 (m, 1H), 2.52-2.50 (m, 1H), $2.31(\mathrm{~s}, 3 \mathrm{H}), 1.86-1.82(\mathrm{~m}, 2 \mathrm{H}), 1.28(\mathrm{t}, 3 \mathrm{H}, \mathrm{J}=7.0$ $\mathrm{Hz}), 1.12(\mathrm{~d}, 3 \mathrm{H}, \mathrm{J}=6.5 \mathrm{~Hz}) ;{ }^{13} \mathrm{C} \mathrm{NMR}\left(500 \mathrm{MHz}, \mathrm{CDCl}_{3}\right) \delta 172.5,81.3,69.2,66.9,65.4$, 60.8, 56.7, 37.9, 28.6, 14.3, 13.3; IR (neat) $\mathrm{cm}^{-1} 3435,2935,2893,2785,1755,1730,1448$, 1340, 1297, 1272, 1207, 1174, 1139, 1125, 1115, 1084, 1055, 1040, 960; HRMS m/z calc. for $\mathrm{C}_{11} \mathrm{H}_{21} \mathrm{O}_{4} \mathrm{~N}\left(\mathrm{M}+\mathrm{H}^{+}\right) 232.1543$, found 232.15375.

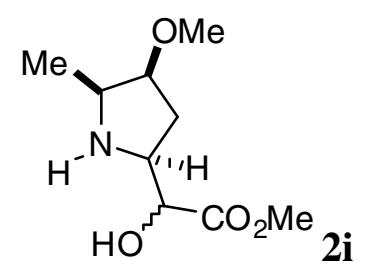

In this case, a second portion of catalyst ( $w / w$ with the substrate) was added to drive the reaction to completion. (Purified by column chromatography on silica gel, ethyl acetate/methanol 9:1, 90\%) ${ }^{1} \mathrm{H}$ NMR $\left(500 \mathrm{MHz}, \mathrm{CDCl}_{3}\right): 4.33(\mathrm{~d}, 0.75 \mathrm{H}, \mathrm{J}=4.0 \mathrm{~Hz}), 4.15$ $(\mathrm{d}, 0.25 \mathrm{H}, \mathrm{J}=3.0 \mathrm{~Hz}), 3.84-3.82(\mathrm{~m}, 3 \mathrm{H}), 3.70-3.59(\mathrm{~m}, 2 \mathrm{H}), 3.37(\mathrm{~s}, 2.25 \mathrm{H}), 3.35(\mathrm{~s}$, $0.75 \mathrm{H}), 3.23-3.18(\mathrm{~m}, 0.25 \mathrm{H}), 3.17-3.13(\mathrm{~m}, 0.75 \mathrm{H}), 2.89$ (broad s, $2 \mathrm{H}), 2.17-1.82(\mathrm{~m}, 2 \mathrm{H})$, $1.24(\mathrm{~d}, 2.25 \mathrm{H}, \mathrm{J}=6.5 \mathrm{~Hz}), 1.21(\mathrm{~d}, 0.75 \mathrm{H}, \mathrm{J}=6.5 \mathrm{~Hz}) ;{ }^{13} \mathrm{C} \mathrm{NMR}\left(500 \mathrm{MHz}, \mathrm{CDCl}_{3}\right) \delta$ 173.9, 173.4, 82.4, 82.2, 71.9, 71.4, 59.0, 58.9, 58.4, 58.1, 57.0, 56.9, 52.3, 52.2, 33.4, 31.7, 14.7, 14.1; IR (neat) $\mathrm{cm}^{-1} 3414,2938,1734,1635,1437,1271,1212,1092,1005,749,649$, $618,581,543$; HRMS $m / z$ calc. for $\mathrm{C}_{9} \mathrm{H}_{17} \mathrm{O}_{4} \mathrm{~N}\left(\mathrm{M}+\mathrm{H}^{+}\right)$204.1230, found 204.12233.

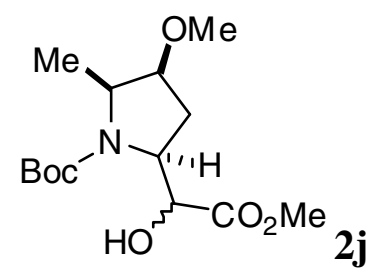

(Purified by column chromatography on silica gel, ethyl acetate/hexane 1:1,95\%) ${ }^{1} \mathrm{H}$ 
NMR (400 MHz, $\mathrm{CDCl}_{3}$ ): 4.85 (broad s, 0.33H), 4.57 (broad s, 0.67H), $4.12(\mathrm{~m}, 1 \mathrm{H}), 4.03$ (m, 1H), 3.80-3.73 (m, 4H), $3.36(\mathrm{~s}, 2 \mathrm{H}), 3.34(\mathrm{~s}, 1 \mathrm{H}), 2.22-1.89(\mathrm{~m}, 2 \mathrm{H}), 1.48(\mathrm{~s}, 6 \mathrm{H})$, $1.46(\mathrm{~s}, 3 \mathrm{H}), 1.15-1.05(\mathrm{~m}, 3 \mathrm{H}) ;{ }^{13} \mathrm{C}$ NMR $\left(500 \mathrm{MHz}, \mathrm{CDCl}_{3}\right) \delta 173.1,155.9,80.7$, 80.5,80.1, 79.4, 79.2, 72.0, 59.5, 58.6, 57.6, 57.4, 56.2, 55.7, 52.2, 30.9, 29.6, 28.4, 14.6, 14.2; IR (neat) $\mathrm{cm}^{-1} 3441,2977,2934,1734,1695,1454,1393,1367,1300,1255,1219$, $1203,1171,1129,1108,1053,1031,1002,859,774 ;$ HRMS $m / z$ calc. for $\mathrm{C}_{14} \mathrm{H}_{25} \mathrm{O}_{6} \mathrm{~N}(\mathrm{M}+$ $\mathrm{Na}^{+}$) 326.1574, found 326.15735 .

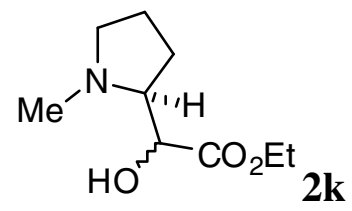

(Purified by column chromatography on silica gel, ethyl acetate, $93 \%){ }^{1} \mathrm{H}$ NMR (400 MHz, $\mathrm{CDCl}_{3}$ ): Diastereomer 1: $4.23(\mathrm{q}, 2 \mathrm{H}, \mathrm{J}=7.0 \mathrm{~Hz}), 3.95(\mathrm{~d}, 1 \mathrm{H}, \mathrm{J}=2.0 \mathrm{~Hz}), 3.41$ (broad s, $1 \mathrm{H}), 3.12(\mathrm{t}, 1 \mathrm{H}, \mathrm{J}=7.0 \mathrm{~Hz}), 2.94-2.91(\mathrm{~m}, 1 \mathrm{H}), 2.42-2.37(\mathrm{~m}, 1 \mathrm{H}), 2.30(\mathrm{~s}, 3 \mathrm{H}), 2.13-2.05$ $(\mathrm{m}, 1 \mathrm{H}), 1.87-1.71(\mathrm{~m}, 3 \mathrm{H}), 1.20(\mathrm{t}, 3 \mathrm{H}, \mathrm{J}=7.0 \mathrm{~Hz})$, Diastereomer $2: 4.34(\mathrm{~d}, 1 \mathrm{H}, \mathrm{J}=3.2$ $\mathrm{Hz}), 4.29$ (q, 2H, J = 7.2 Hz), 3.18-3.14 (m, 1H), 2.77-2.72 (m, 1H), 2.43 (s, 3H), 2.41-2.32 $(\mathrm{m}, 1 \mathrm{H}), 1.85-1.72(\mathrm{~m}, 4 \mathrm{H}), 1.33(\mathrm{t}, 3 \mathrm{H}, \mathrm{J}=7.2 \mathrm{~Hz}) ;{ }^{13} \mathrm{C} \mathrm{NMR}\left(500 \mathrm{MHz}, \mathrm{CDCl}_{3}\right) \delta$ Diastereomer 1: 174.1, 71.9, 67.1, 61.0, 58.0, 42.0, 30.7, 24.2, 14.2, Diastereomer 2: 172.5, 68.7, 67.0, 61.0, 57.0, 40.0, 24.6, 23.1, 14.2; IR (neat) $\mathrm{cm}^{-1} 3466,3351,2951,2790,1730$, $1457,1261,1204,1126,1111,1028,885$; HRMS $m / z$ calc. for $\mathrm{C}_{9} \mathrm{H}_{17} \mathrm{O}_{3} \mathrm{~N}\left(\mathrm{M}+\mathrm{H}^{+}\right)$ 188.1281, found 188.12865 .<smiles>CCC1CCN(C)C1C(O)C(OC)OC</smiles>

(Purified by column chromatography on silica gel, ethyl acetate, 93\%) ${ }^{1} \mathrm{H}$ NMR (400 MHz, $\left.\mathrm{CDCl}_{3}\right): 4.35(\mathrm{~d}, 1 \mathrm{H}, \mathrm{J}=3.6 \mathrm{~Hz}), 3.80(\mathrm{~s}, 3 \mathrm{H}), 3.77(\mathrm{~d}, 1 \mathrm{H}, \mathrm{J}=6.4 \mathrm{~Hz}), 3.16-3.12(\mathrm{~m}, 1 \mathrm{H})$, $2.93\left(\mathrm{dd}, 1 \mathrm{H}, \mathrm{J}_{1}=3.2 \mathrm{~Hz}, \mathrm{~J}_{2}=8.8 \mathrm{~Hz}\right), 2.44(\mathrm{~s}, 3 \mathrm{H}), 2.40-2.34(\mathrm{~m}, 1 \mathrm{H}), 2.13-2.05(\mathrm{~m}, 1 \mathrm{H})$, 1.94-1.88 (m, 1H), 1.54-1.40 (m, 3H), $0.86(\mathrm{t}, 3 \mathrm{H}, \mathrm{J}=7.2 \mathrm{~Hz}) ;{ }^{13} \mathrm{C} \mathrm{NMR}\left(500 \mathrm{MHz}, \mathrm{CDCl}_{3}\right)$ $\delta$ 173.2, 70.1, 69.6, 55.3, 52.1, 43.5, 41.0, 30.8, 22.7, 13.1; IR (neat) $\mathrm{cm}^{-1} 3482,2960,2933$, 2874, 2786, 1736, 1449, 1207, 1144, 1069, 1034, 736, 624, 582; HRMS $m / z$ calc. for $\mathrm{C}_{10} \mathrm{H}_{19} \mathrm{O}_{3} \mathrm{~N}\left(\mathrm{M}+\mathrm{H}^{+}\right)$202.1438, found 202.14311.

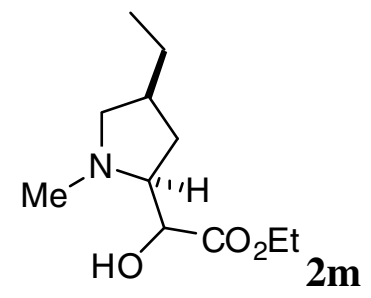

(Purified by column chromatography on silica gel, ethyl acetate, 92\%) ${ }^{1} \mathrm{H}$ NMR (400 MHz, 
$\left.\mathrm{CDCl}_{3}\right): 4.36(\mathrm{~d}, 1 \mathrm{H}, \mathrm{J}=3.2 \mathrm{~Hz}), 4.30(\mathrm{q}, 2 \mathrm{H}, \mathrm{J}=7.2 \mathrm{~Hz}), 2.93\left(\mathrm{dd}, 1 \mathrm{H}, \mathrm{J}_{1}=4.0 \mathrm{~Hz}, \mathrm{~J}_{2}=\right.$ $9.6 \mathrm{~Hz}), 2.84-2.79(\mathrm{~m}, 1 \mathrm{H}), 2.60(\mathrm{t}, 1 \mathrm{H}, \mathrm{J}=8.8 \mathrm{~Hz}), 2.39(\mathrm{~s}, 3 \mathrm{H}), 2.04-1.97(\mathrm{~m}, 1 \mathrm{H})$, $1.89-1.82(\mathrm{~m}, 1 \mathrm{H}), 1.53-1.39(\mathrm{~m}, 3 \mathrm{H}), 1.35(\mathrm{t}, 3 \mathrm{H}, \mathrm{J}=7.2 \mathrm{~Hz}), 0.91(\mathrm{t}, 3 \mathrm{H}, \mathrm{J}=7.2 \mathrm{~Hz}) ;{ }^{13} \mathrm{C}$ NMR (500MHz, $\left.\mathrm{CDCl}_{3}\right) \delta 172.5,67.8,67.7,61.8,61.0,39.8,37.2,31.1,28.5,14.2,12.5$; IR (neat) $\mathrm{cm}^{-1} 3420,2958,1734,1695,1653,1613,1559,1458,1219,1130,1031,654$, 609, 521; HRMS $m / z$ calc. for $\mathrm{C}_{11} \mathrm{H}_{21} \mathrm{O}_{3} \mathrm{~N}\left(\mathrm{M}+\mathrm{H}^{+}\right)$216.1594, found 216.15918 .

\section{Intermediates isolated during mechanistic studies}<smiles>CCOC(=O)C(O)c1cc(OC)c2n1CCCC2</smiles>

(Purified by column chromatography on silica gel, ethyl acetate/hexane 1:1, 64\%, 0.12 mmol) ${ }^{1} \mathrm{H}$ NMR $\left(400 \mathrm{MHz}, \mathrm{CDCl}_{3}\right) \delta 5.82(\mathrm{~s}, 1 \mathrm{H}), 5.16(\mathrm{~s}, 1 \mathrm{H}), 4.33-4.25(\mathrm{~m}, 2 \mathrm{H})$, 3.94-3.87 (m, 2H), $3.71(\mathrm{~s}, 3 \mathrm{H}), 2.70-2.66(\mathrm{~m}, 2 \mathrm{H}), 1.95-1.92(\mathrm{~m}, 2 \mathrm{H}), 1.81-1.76(\mathrm{~m}, 2 \mathrm{H})$, $1.41(\mathrm{t}, 3 \mathrm{H}, \mathrm{J}=7.2 \mathrm{~Hz}) ;{ }^{13} \mathrm{C}$ NMR $\left(500 \mathrm{MHz}, \mathrm{CDCl}_{3}\right) \delta 172.9,142.2,123.2,115.5,95.7$, 66.1, 62.1, 58.8, 42.9, 23.6, 20.6, 20.4, 14.1; IR (neat) $\mathrm{cm}^{-1} 3435,2934,2856,1752,1732$, $1451,1372,1347,1292,1267,1199,1142,1126,1095,1038$; HRMS $\mathrm{m} / \mathrm{z}$ calc. for $\mathrm{C}_{13} \mathrm{H}_{19} \mathrm{O}_{4} \mathrm{~N}\left(\mathrm{M}^{+}\right)$253.1309, found 253.13101.

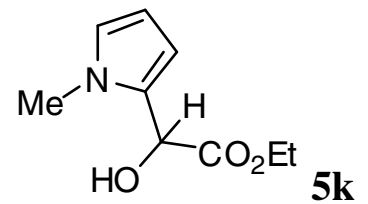

(Purified by column chromatography on silica gel, ethyl acetate/hexane 1:1, 99\%, 0.31 mmol) ${ }^{1} \mathrm{H} \mathrm{NMR}\left(500 \mathrm{MHz}, \mathrm{CDCl}_{3}\right) \delta 6.62(\mathrm{t}, 1 \mathrm{H}, \mathrm{J}=2.0 \mathrm{~Hz}), 6.06(\mathrm{~d}, 2 \mathrm{H}, \mathrm{J}=2.0 \mathrm{~Hz}), 5.21$ $(\mathrm{d}, 1 \mathrm{H}, \mathrm{J}=6.5 \mathrm{~Hz}), 4.34-4.26(\mathrm{~m}, 2 \mathrm{H}), 3.67(\mathrm{~s}, 3 \mathrm{H}), 3.06(\mathrm{~d}, 1 \mathrm{H}, \mathrm{J}=7.0 \mathrm{~Hz}), 1.30(\mathrm{t}, 3 \mathrm{H}, \mathrm{J}$ $=7.0 \mathrm{~Hz}) ;{ }^{13} \mathrm{C}$ NMR $\left(500 \mathrm{MHz}, \mathrm{CDCl}_{3}\right) \delta 172.8,129.1,124.1,108.4,107.0,66.4,62.2$, 34.1, 14.1; IR (neat) $\mathrm{cm}^{-1} 3427,2979,1732,1486,1444,1416,1301,1262,1209,1151$, 1090, 1056, 1017, 715; HRMS $m / z$ calc. for $\mathrm{C}_{9} \mathrm{H}_{13} \mathrm{O}_{3} \mathrm{~N}\left(\mathrm{M}+\mathrm{Na}^{+}\right) 206.0788$, found 206.07820. 